\title{
Immune Cell Composition of Liver Hepatocellular Carcinoma and its clinical relevance: A research based on TCGA database
}

\section{Yucheng Ji}

Shanghai Jiao Tong University School of Medicine

Guang-xiang Gu ( $\nabla$ guguangxiang@163.com )

https://orcid.org/0000-0002-9585-5284

\section{Research article}

Keywords: TCGA; Liver Hepatocellular carcinoma (LIHC); Immune cells; Overall survival

Posted Date: October 4th, 2019

DOl: https://doi.org/10.21203/rs.2.15624/v1

License: (c) (1) This work is licensed under a Creative Commons Attribution 4.0 International License.

Read Full License 


\section{Abstract}

Background Liver hepatocellular carcinoma (LIHC), as the main type of liver cancer, has become a main health issue as the third-most common cause of mortality in cancer patients. However, conventional chemo- or radio- therapies shows little improvement in survival, which calls for novel therapies. Because of the immunotolerance mechanism existing naturally in liver, immunotherapy provides significant effect in treatment of LIHC patients. Up to now, various immunotherapies have been proposed, but due to the complex pathways from which LIHC cancerous cell escape immunosurveillance, combined therapies are often needed, which are still under development. Methods In the current study, with data downloaded from TCGA database, CIBERSORT was performed for identifying the composition of infiltrating immune cells and further statistical analyses using R 3.5.3 were carried out, aiming at connecting specific immune cells with clinical survival. Results With data of immune and stromal scores downloaded from the website of MD Anderson Cancer Centre, both showed significance in survival time. Further analyses based on the result of CIBERSORT demonstrated that the number of macrophages M0 and T cells CD8 infiltration between para-carcinoma and tumour tissues are markedly different. With combination of clinical data, we were able to identify that a higher amount of activated NK cells $(p=0.008)$ and a lower amount of resting NK cells $(p=0.047)$ presented a longer survival time. Conclusion With the help of the TCGA database and multiple techniques, statistical analyses of transcriptome profiling data and clinical data were successfully carried out. The results in this study may pave the way for a new therapeutic strategy which could be combined with current treatments to further improve the clinical outcome of LIHC patients. Further and deeper investigation of other available data, however, were needed in order to verify the results of current study.

\section{Background}

Liver cancer has become a main health issue throughout the world. According to the statistics available in GLOBOCAN database(1), despite relatively low incidence of liver cancer overall compared with breast or lung cancer in 2018 , its mortality rate was at the fourth place. In males, especially, the second-most cancer-related death were from liver cancer (Figure 1).

Among all types of liver cancer, liver hepatocellular carcinoma (LIHC) is the most common one (2), whereas most patients fail to be detected early enough and are reduced to palliative therapy (3). Because of the immunotolerance mechanism existed naturally in liver, cancer cells can easily escape from the immunosurveillance system, and such tolerance is further enhanced in patients with hepatocellular carcinoma according to previous studies $(4,5)$. As a result, immunotherapy provides significant effect in treatment of LIHC patients, while traditional therapies such as chemotherapy show no benefit for survival rate (6).

Furthermore, increasing researches (7) have proven that tumour microenvironment in LIHC, which mainly includes fibroblasts, immune cells and endothelial cells, plays an important role in pathogenesis and prognosis. The algorithm developed by Yoshihara et al. (8) helps calculate immune and stromal scores 
which indicate immune and stromal cells infiltration respectively, which then helps interpret the association between tumour microenvironment and cancer outcomes.

In recent years, novel immunotherapies like anti-CTLA-4 and anti-PD-1 have been proposed (9), and at the same time have longer durability of response and lower cytotoxicity (10). Due to the complex pathways $\mathrm{LIHC}$ cancerous cell escape from immunosurveillance, combined therapies are often needed. Thus, this article presents results of immune cells infiltration in LIHC patients and relationship between specific immune cells and clinical survival. Hopefully, the identification of related immune cells would pave the way for a novel combined strategy of various immunotherapies against LIHC.

\section{Methods}

\section{Patients and samples}

All transcriptome profiling files and clinical data of 376 patients with LIHC were downloaded from TCGA database (11). The results of immune cells in each sample were performed on the website of CIBERSORT developed by Newman et al (12), and those with a p-value less than 0.05 were selected for further study. The results of Stromal and Immune scores were downloaded from the website of MD Anderson Cancer Centre (13) using the ESTIMATE algorithm (8).

\section{Statistical analysis}

All analyses were performed with R 3.5.3. Wilcoxon test was used to define the correlations between clinical status and immune data. Survival analysis was performed using Kaplein-Meier curve and log-rank test. Other necessary tests are performed with the help of R packages (Figure 2).

\section{Results}

\section{Stromal and Immune scores show correlation with patients' survival}

Within all the patients with complete clinical data, 121(32.6\%) were female, and 250(67.4\%) were male. Clinical staging revealed 171(46.1\%) Stage I, 86(23.2\%) Stage II, 85(22.9\%) Stage III, 5(1.3\%) Stage IV and $24(6.5 \%)$ unknown. With data downloaded from the website of MD Anderson Cancer Centre, it showed that stromal score ranged from -1741.56 to 1195.07 , while, immune scores ranged from -1209.16 to 2934.36 .

In order to determine whether there was correlation between survival time and stromal or immune scores, we divided the patients into two groups based on scores: the lowest $25 \%$ as 'low' and the rest $75 \%$ as 'high'. Both Kaplein-Meier curve (K-M curve) and log-rank test showed stromal and immune scores were significantly co-related with patients' survival. (Figure 3a \& 3b) 


\section{Defining immune cells composition in acquired TCGA LIHC transcriptome profiles}

As shown in figure $3 a \& 3 b$, a high percentage of immune cells infiltration in tumour tissues provides long survival time, which is in accordance with evidence previously reported (14). Therefore, we suggested that immune cells play an important role in LIHC progression and prognosis. To find out specific immune cells composition for further analyses, CIBERSORT (Newman et al.) was carried out using R script downloaded from its website. Results were selected if $P$ value was less than 0.05 , and were presented with heatmap and correlation heatmap of 22 types of immune cells (Figure $4 a \& 4 b$ ).

\section{Immune cell related survival analyses}

We combined clinical data with immune cell profiles and carried out survival analyses, producing K-M curves for each type of immune cells. However, neither macrophages M0 nor T cells CD8 showed significant correlation with survival time $(p>0.05)$. Interestingly, when samples with a high amount of activated NK cells presented a longer survival time $(p=0.008)$, and samples with a low amount of resting NK cells exactly presented a shorter survival time $(p=0.047$; Figure5 (a)-(d)). This indicates that the activity of NK cells in tumour tissues may have a great impact on the prognosis of patients, which is not clearly shown previously.

\section{Discussion}

In the current study, we identified macrophages M0 and T cells CD8 as the two mostly infiltrated cells in $\mathrm{LIHC}$, and further analyses demonstrated a negative relationship between them, which meant LIHC patients could be divided into either macrophages-M0-dominated or T-cells-CD8-dominated. Thus, survival analysis was carried out, with a surprising result that neither cell showed significant influence on the survival time. Interestingly, the NK cells pairs, activated and resting NK cells, obviously affect prognosis, suggesting a possible role of NK cells in eliminating malignant tumour cells. However, considering the relatively small number of cases involved and the low amount of cell counts as shown in Figure4 (a), more repeated analyses with disparate samples should be performed in the future.

As the third-most common cause of mortality in cancer patients (15), LIHC responds poorly to conventional chemotherapy, which calls for novel therapeutics. So far, new drug development mainly focused on $T$ cells and B cells, which are the two primary composing cells of immune repertoire, serving as potential biomarkers for the diagnosis and treatment targets for LIHC. (16) Unfortunately, neither of them demonstrated significance in the current study using TCGA database, but the valid results of NK cells corresponded with what Juengpanich et al reviewed (17): therapies based on NK cells can subsequently lead to higher survival rate of HCC patients. Moreover, the completely opposite results that higher activated NK cells are associated with longer survival time, while higher resting NK cells with 
shorter time, provides possibility for developing combined treatment which includes transforming NK cells into active forms.

The main limitation of this study lies in the limited number of valid cases (with $p<0.05$ ) after applying the CIBERSORT algorithm. Possible reasons include diversity of cell infiltration in LIHC and insufficient number of cases for a more accurate CIBERSORT prediction. Hence, further studies should include other available databases and/or real-time clinical data.

\section{Abbreviations}

LIHC: liver hepatocellular carcinoma; NK cell: natural killer cell;

\section{Declarations}

\section{Acknowledgements}

Not applicable

\section{Authors' contributions}

Yu-cheng Ji performed data selection, analysis and was a contributor in writing the manuscript. Guangxiang Gu helped interpret results, advise appropriate statistical approach and proper language use.

\section{Funding}

Not applicable

\section{Availability of data and materials}

All data used are included in this manuscript.

\section{Ethics approval and consent to participate}

Not applicable (using open-access online database) 
Not applicable

\section{Competing interests}

The authors declare that they have no competing interests.

\section{References}

1. The GLOBOCAN database. http://gco.iarc.fr/. Accessed 20 April 2019

2. Llovet JM, Zucman-Rossi J, Pikarsky E, Sangro B, Schwartz M, Sherman M, et al. Hepatocellular carcinoma. Nat Rev Dis Prim. 2016;2:16018

3. Roth GS, Decaens T. Liver immunotolerance and hepatocellular carcinoma: Patho-physiological mechanisms and therapeutic perspectives. European Journal of Cancer. 2017; 87:101-112

4. Wan S, Zhao E, Kryczek I, Vatan L, Sadovskaya A, Ludema G, Simeone DM, Zou W, Welling TH. Tumor-associated macrophages produce interleukin 6 and signal via STAT3 to promote expansion of human hepatocellular carcinoma stem cells. Gastroenterology. 2014;147(6):1393-404

5. Mizukoshi and Kaneko. Immune cell therapy for hepatocellular carcinoma. Journal of Hematology \& Oncology. 2019; $12: 52$

6. Llovet JM, Hernandez-Gea V. Hepatocellular carcinoma: reasons for phase III failure and novel perspectives on trial design. Clin Cancer Res. 2014;20(8):2072-9.

7. Novikova M. V., Khromova N. V., and Kopnin P. B. Components of the Hepatocellular Carcinoma Microenvironment and Their Role in Tumor Progression. Biochemistry (Moscow). 2017; Vol. 82, No. 8, pp. 861-873.

8. Yoshihara K, Shahmoradgoli M, Martínez E, Vegesna R, Kim H, Torres-Garcia W, Treviño V, Shen H, Laird PW, Levine DA, Carter SL, Getz G, Stemke-Hale K, et al. Inferring tumour purity and stromal and immune cell admixture from expression data. Nat Commun. 2013; 4:2612.

9. Prieto J, Melero I, Sangro B. Immunological landscape and immunotherapy of hepatocellular carcinoma. Nat Rev Gastroenterol Hepatol. 2015;12(12):681-700.

10. Joan M. C. Bull. A review of immune therapy in cancer and a question:can thermal therapy increase tumor response? International Journal of Hyperthermia. 2018; 34:6, 840-852

11. The TCGA database. https://portal.gdc.cancer.gov/repository. Accessed 10 March 2019

12. CIBERSORT. https://cibersort.stanford.edu/. Accessed 15 March 2019

13. MD Anderson Cancer Centre. https://bioinformatics.mdanderson.org/estimate/index.html. Accessed 15 April 2019

14. Galon J, Pagès F, Marincola FM, Thurin M, Trinchieri G, Fox BA, Gajewski TF, Ascierto PA. The Immune Score as a New Possible Approach for the Classification of Cancer. J Transl Med. 2012; 10:1. 
15. Bertino G, Demma S, Ardiri A, Proiti M, Mangia A, Gruttadauria S, Toro A, Di Carlo I, Malaguarnera G, Bertino N, Malaguarnera M. The immune system in Hepatocellular carcinoma and potential new immunotherapeutic strategies. Biomed Res Int. 2015; 2015:731469.

16. Han YX, Li HM, Guan YF, Huang J Immune repertoire: A potential biomarker and therapeutic for hepatocellular carcinoma. Cancer Letters. 2016; 379:206-212

17. Juengpanich S, Shi L, Iranmanesh Y, Chen J, Cheng ZZ, Khoo KA, Pan L, Wang YF and Cai XJ. The role of natural killer cells in hepatocellular carcinoma development and treatment: A narrative review. Translational Oncology. 2019; 12:1092-1107

\section{Figures}

Estimated age-standardized incidence and mortality rates (World) in 2018, worldwide, males, all ages

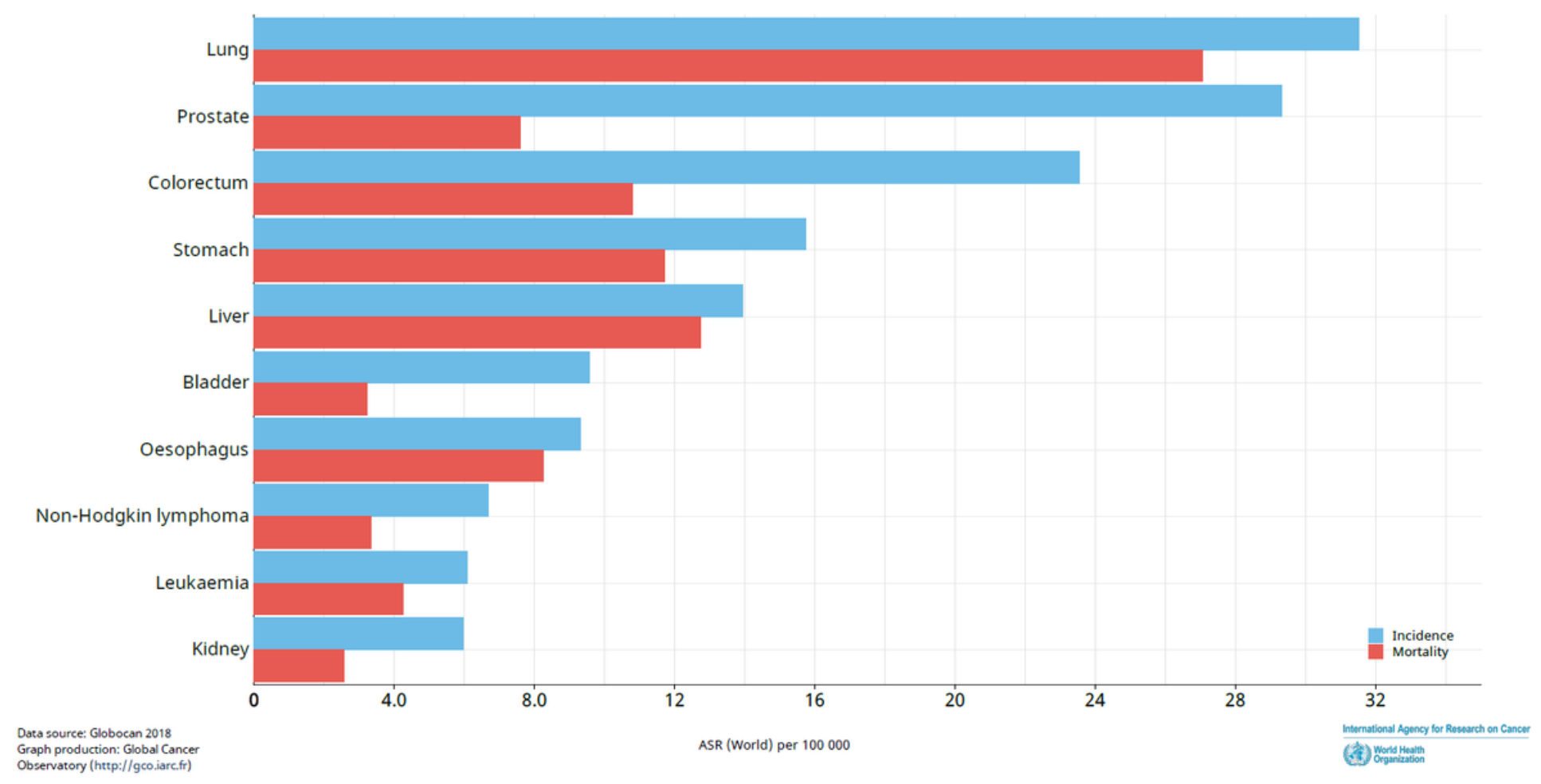

\section{Figure 1}

Estimated age-standardized cancer incidence and mortality rates (World) in 2018 in males of all ages 


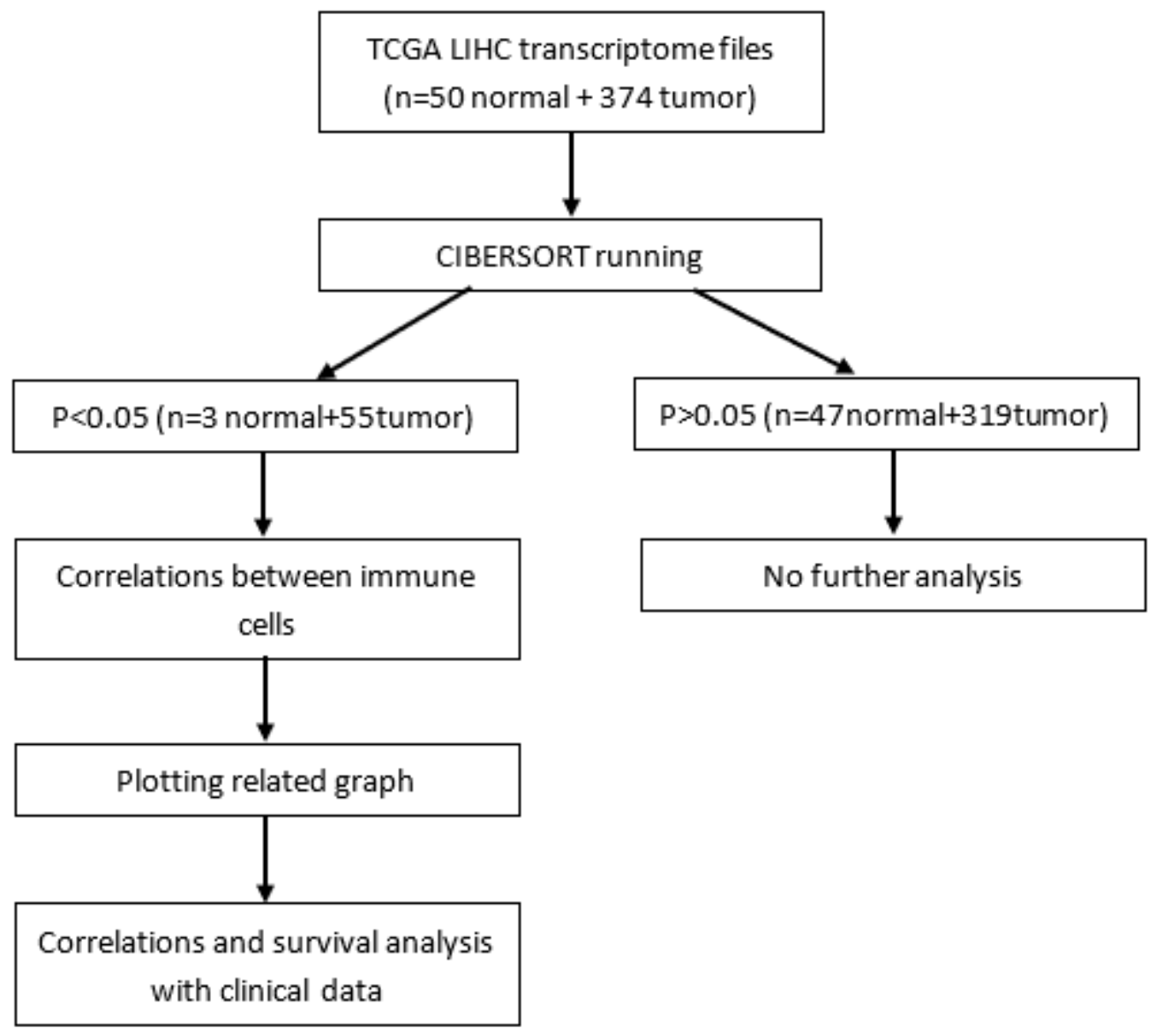

Figure 2

Flow chart of the main analysis procedures in this study
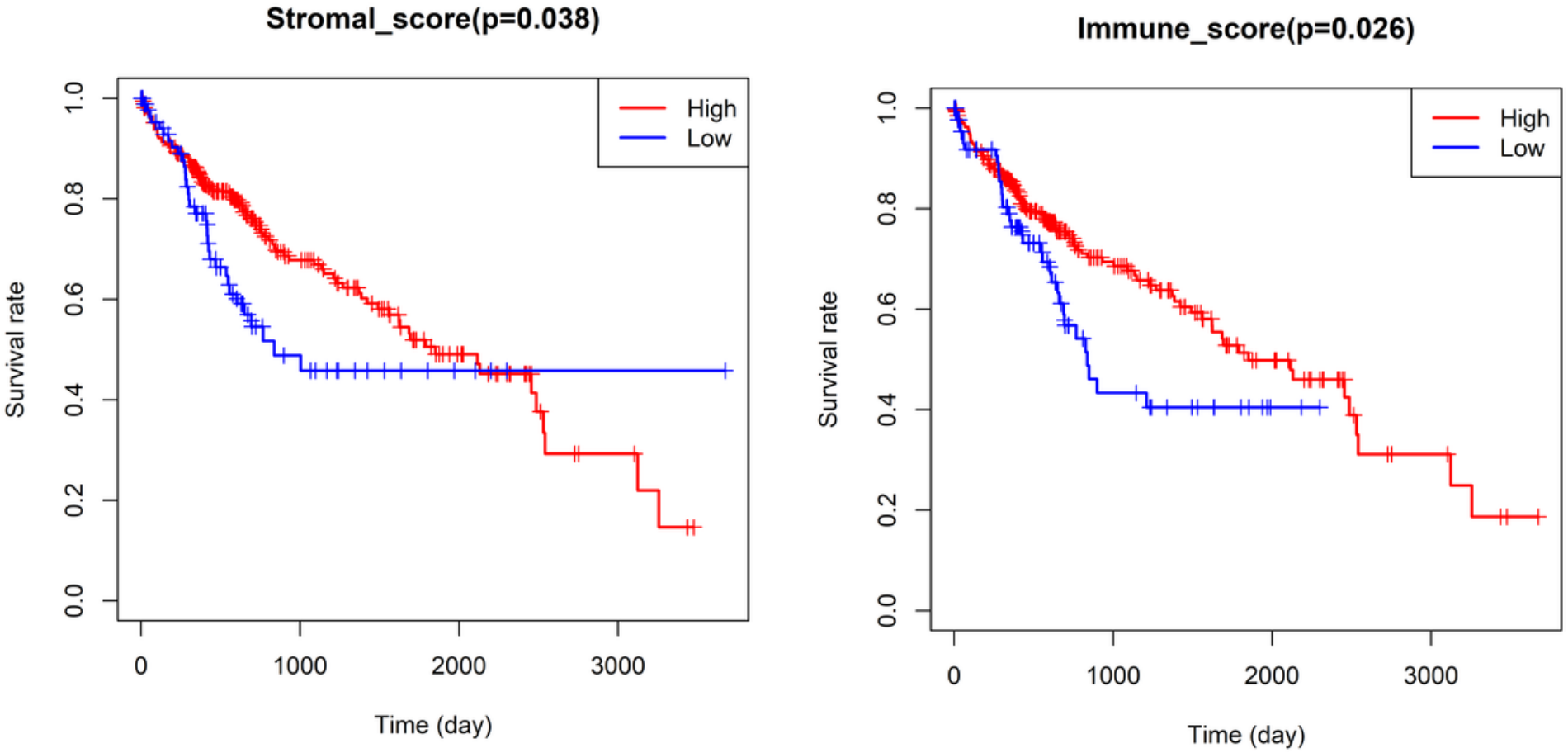

Figure 3 
(a) showed the result of Kaplein-Meier test. Patients were divided into two groups, the lowest $25 \%$ as 'low' and the rest $75 \%$ as 'high'. The curve shows that mean survival time of 'high' group is longer than the 'low' group ( 587.5 days vs 421 days, $p=0.038$ ); (b) showed the result of Kaplein-Meier test. Patients were also divided into two groups, the lowest $25 \%$ as 'low' and the rest $75 \%$ as 'high'. The curve shows that mean survival time of 'high' group is longer than the 'low' group ( 577 days vs 428 days, $p=0.026$ )

\section{Figure 4}

. (a) Heatmap of immune cell composition of 58 samples whose $p$ value is less than $0.05,3$ of which are para-carcinoma tissues (indicated as blue blocks in the top bar) and 55 of which are tumour tissues (indicated as pink blocks in the top bar). While most cells present no significant difference, the number of macrophages $\mathrm{M} 0$ and $\mathrm{T}$ cells CD8 infiltration between para-carcinoma and tumour tissues are markedly different.(b) Correlation heatmap of the 22 types of immune cells. T cells follicular helper and T cells CD8, T cells CD4 memory activated and T cells CD8 were the two most positively correlated pairs, while macrophage $\mathrm{M} 0$ and $\mathrm{T}$ cells $\mathrm{CD} 8, \mathrm{~T}$ cells $\mathrm{CD} 4$ memory resting and activated were the two most negatively correlated pairs. 
Macrophages $M O(p=0.249)$
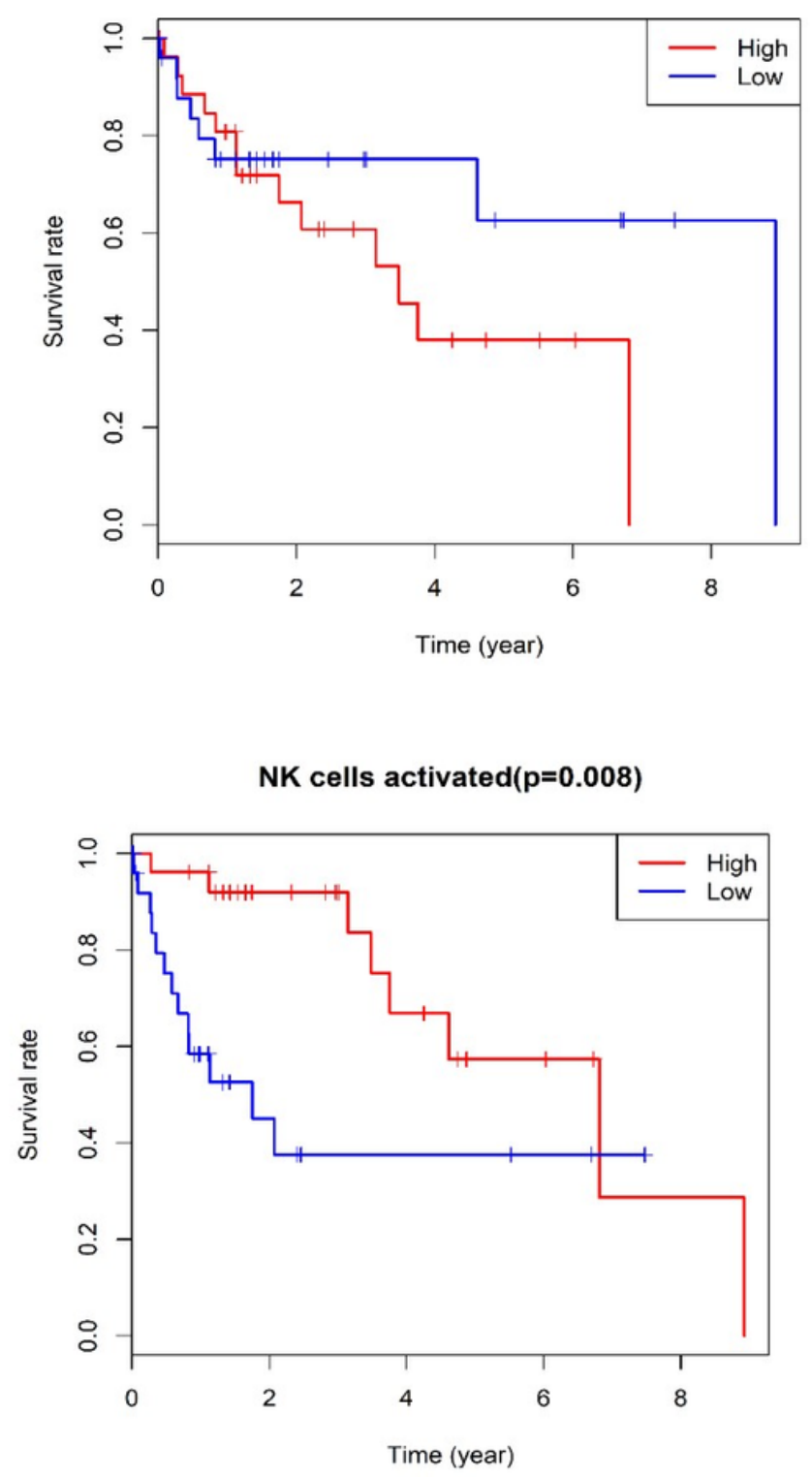

T cells $\operatorname{CD} 8(p=0.076)$

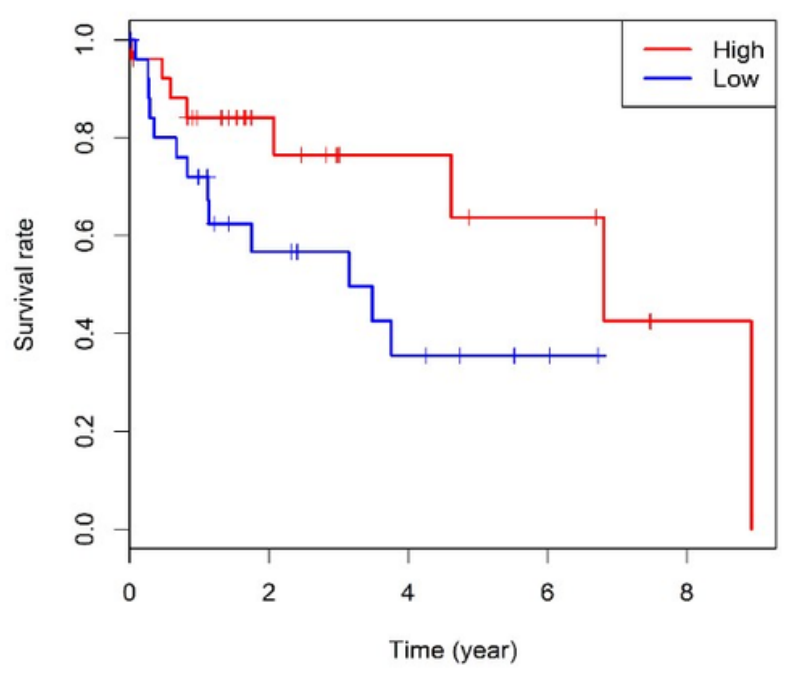

NK cells resting $(p=0.047)$

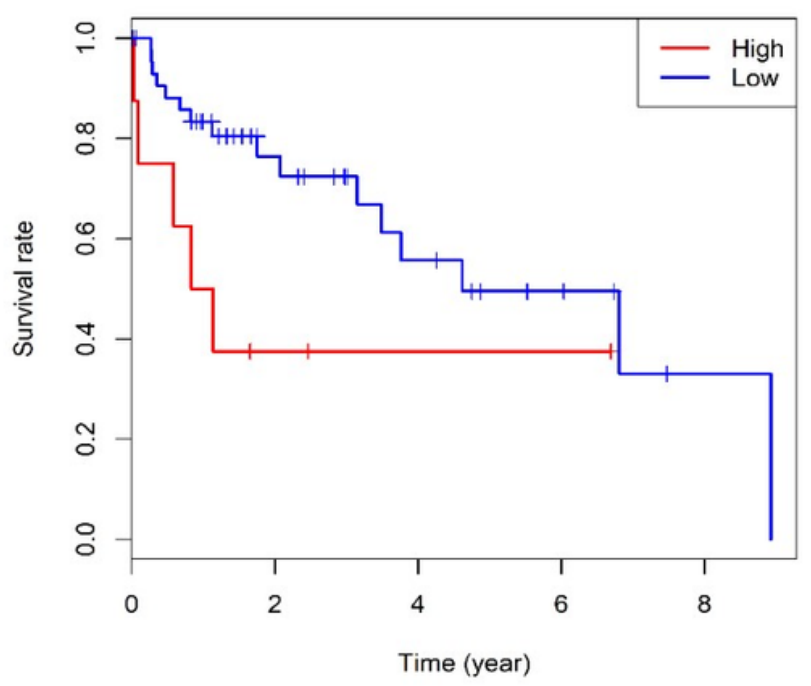

Figure 5

(a)-(d) show K-M curves of four types of immune cells divided by medians into high and low groups. Surprisingly, though macrophage M0 and T cells CD8 both show obvious difference between paracarcinoma tissues and tumour tissues, correlations with survival time were not as significant as predicted. But interestingly, activated and resting NK cells expressed impact on survival status. 